\title{
Asymmetric Early Post-Magma Ocean Crust Building on the Moon's Nearside
}

\author{
STEPHEN M. Elardo ${ }^{1}$, MATTHIEU LANEUVILlE ${ }^{2}$, \\ FRANCIS M. MCCUBBIN ${ }^{3}$, AND CHARLES K. SHEARER ${ }^{4}$ \\ ${ }^{1}$ Department of Geological Sciences, University of Florida \\ ${ }^{2}$ Earth-Life Science Institute, Tokyo Institute of Technology \\ ${ }^{3}$ NASA - Johnson Space Center \\ ${ }^{4}$ Institute of Meteoritics, University of New Mexico
}

The Moon's crust is hemispherically asymmetric in terms of crustal thickness, the composition of the primary lunar magma ocean- (LMO) derived anorthosite, and in the distribution of both geochemically incompatible elements (including the radioactive elements $\mathrm{K}, \mathrm{U}$, and $\mathrm{Th}$ ) and erupted lavas. The concentration of radioactive elements on the nearside in the Procellarum KREEP Terrane (PKT) is widely thought to have provided heat for mantle melting from $\sim 3.9$ $\mathrm{Ga}$ to $\sim 1 \mathrm{Ga}$. However, a link between KREEP and the earliest pulse of post-differentiation crust building, represented by the Mg-suite, is highly debated.

Here we assess whether Mg-suite crust-building magmatism was a global or regional event using hightemperature experiments and thermal evolution calculations. Our high-temperature, 1-atm experiments simulate Mg-suite magma production from a hybridized source region [1] consisting of deep mantle dunites, crustal anorthosite, and variable amounts of KREEP from $0-50$ wt. \%. Our results show that the change in bulk chemical composition due to increasing amounts of KREEP lowers the melting point of the three-component system dramatically. When we consider only experimentally produced melts that match the major and trace element compositions of $\mathrm{Mg}$-suite parental melts estimated from samples, we conclude that there may have been between $\sim 4-13$ times more $\mathrm{Mg}$-suite melt production in and around the PKT post-LMO relative to the farside.

We also conducted cooling calculations for the Mg-suite source regions of the same compositions to assess the effects of radiogenic heating. Using lower crustal cooling rates of 0.2 $-2.5 \mathrm{~K} / \mathrm{Myr}$ from [2], all $\mathrm{Mg}$-suite source regions containing $>25 \%$ KREEP increase in temperature regardless of cooling rate. Conversely, all $\mathrm{Mg}$-suite magma sources with $<5 \%$ KREEP, as would be expected on the farside, cool down with time. The combined effects of KREEP-induced depression of mantle melting temperatures and the heat produced from radioactivity strongly suggest that $\mathrm{Mg}$-suite crust-building was concentrated on the nearside in and around the PKT.

[1] Elardo et al. (2011) GCA, 75, $3024-3045$

[2] Laneuville et al. (2018) JGR-P, 123, $3144-3166$ 